\title{
A new way to measure canine welfare
}

Animal welfare science focuses on assessing and maximizing animals' quality of life. In the research context, improving the welfare of laboratory animals is a high priority. To this end, objective measures of animal welfare are greatly needed. One way to evaluate welfare is to consider an animal's affective state, which can be assessed indirectly by observing its influence on cognitive processes, such as those underlying the interpretation of ambiguous signals (or judgment bias). A negative affective state leads to an expectation of negative outcomes and thus a negative bias in the interpretation of ambiguous signals (a pessimistic outlook). In contrast, a positive affective state leads to an expectation of positive outcomes and a positive bias in the interpretation of ambiguous signals (an optimistic outlook). A recent report in PLoS One $(9$, e107794; 2014) describes the use of a portable, automated apparatus to train and test the judgment bias of dogs. "This research is exciting because it measures positive and negative emotional states in dogs objectively and non-invasively. It offers researchers and dog owners an insight into the outlook of dogs and how that changes,"

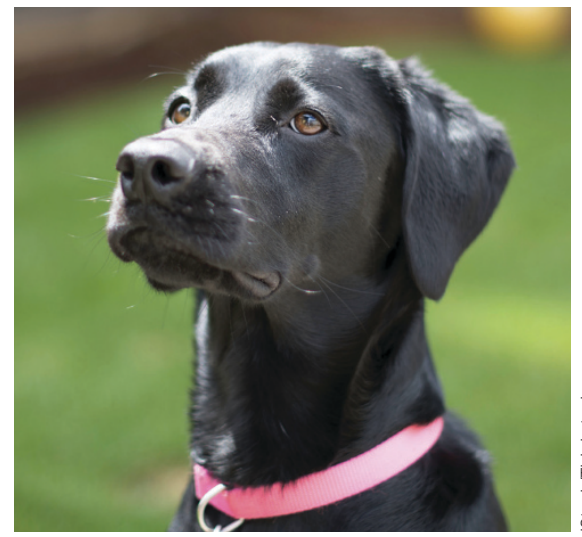

said Melissa Starling (University of Sydney, Australia), who led the research, in a press release. "Finding out as accurately as possible whether a particular dog is optimistic or pessimistic is particularly helpful in the context of working and service dogs and has important implications for animal welfare."

Dogs were taught to differentiate between two different sounds (two octaves apart), one associated with access to a small volume of lactose-free milk (a reward) and the other not associated with a reward. Once they learned to discriminate between the two notes, they were presented with ambiguous tones and their responses were measured. Some dogs responded to the ambiguous tones, even those that were more similar to the unrewarded sound than to the sound associated with milk. Other dogs did not respond to the ambiguous tones. The results indicate that judgment bias exists in dogs, differs between dogs and can be measured objectively as a readout of affective state.

Starling explained some applications of her work. "This research could help working dog trainers select dogs best suited to working roles. [...] A pessimistic dog that avoids risks would be better as a guide dog while an optimistic, persistent dog would be more suited to detecting drugs or explosives. [...] This research has the potential to completely remodel how animal welfare is assessed. If we know how optimistic or pessimistic an animal usually is, it's possible to track changes in that optimism that will indicate when it is in a more positive or negative emotional state than usual. [...] It could be used to monitor their welfare in any environment, to assess how effective enrichment activities might be in improving welfare, and pinpoint exactly what a dog finds emotionally distressing."

\section{CIRCULATING TUMOR CELISS CAPTURED FOR ANAIYSIS}

Circulating tumor cells (CTCs) are shed from primary tumors into the bloodstream, mediating the spread of cancer to other organs.

Little is known about these cells, partly because they are rare ( 1-10 tumor cells per 10 billion normal blood cells in $1 \mathrm{ml}$ of blood) and difficult to isolate for molecular analysis.

David Ting (Massachusetts General Hospital (MGH) Cancer Center, Boston) and his team have previously attempted to study pooled CTCs enriched from the blood, but this approach only makes it possible to identify the most highly expressed genes in the pooled cells and cannot be used to distinguish genetic differences within the cell population.

But now Ting and his colleagues have developed a new technique for isolating CTCs for transcriptional analysis. They demonstrate this technique in a new study, published in Cell Reports $(8,1905-1918 ; 2014)$. The researchers isolated 168 single CTCs from the bloodstreams of five pancreatic tumor-bearing mice. Of these cells, 75 had RNA of sufficient quality to proceed with next-generation RNA sequencing. The researchers noted that the remaining cells with damaged RNA were likely nonviable cancer cells and that the damage was not the result of the CTC isolation process. Ting's team compared the genome-wide expression profiles of these viable CTCs with matched primary tumors from mouse models as well as human CTCs of pancreatic, breast and prostate cancers. Three different subsets of CTCs were identified, all of which were distinct from the sequences of the mouse primary tumors and human cancer cell lines. Extracellular matrix genes, which are important for the establishment of metastases, were highly expressed in the CTCs.

The device developed by Ting's team, called the CTC-iChip, enables the isolation of all CTCs in a blood sample using a process called epitope-independent microfluidic capture. The first step was hydrodynamic size-based separation of all nucleated cells away from red blood cells, platelets and plasma. The next step of the process excluded the vast majority of white blood cells from the sample so that only CTCs remained. The advantage of this approach is that it leaves the CTCs in solution, rendering them more suitable for advanced RNA sequencing techniques to reveal the gene expression patterns of each individual cell.

Said Ting in a press release, "Our ability to combine a novel microfluidic CTC isolation device, developed here at MGH, with single-cell RNA sequencing has given us new biological insights into these cells and revealed novel avenues to try and block the spread of cancer." 